\title{
Le contrat de travail pour assistante médicale
}

\author{
N. Reichen
}

\section{Introduction}

Lorsqu'un médecin exploite son cabinet médical à titre indépendant, il doit en général engager et salarier une ou plusieurs assistantes médicales (cette notion regroupe les assistants médicaux de sexe masculin que l'on pourrait rencontrer, le contrat type mis en place par la FMH et les organisations de salariés FSAAM et SVA étant intitulé «contrat de travail pour assistante médicale»). Le présent article abordera différents droits et devoirs liés à ce rapport contractuel, ainsi que certains points connexes méritant d'être soulevés.

\section{Le contrat de travail}

Le lien unissant l'assistante médicale au praticien indépendant est de nature contractuelle, impliquant une certaine continuité dans les relations de travail et un rapport de subordination. C'est donc un contrat de travail qui lie le médecin et son assistante. Les règles du Code des obligations sur ce type de contrat s'appliquent donc.

Il faut différencier le cas où un médecin ferait appel à l'un de ses pairs pour une tâche en général définie. Quand bien même les deux praticiens collaborent fréquemment, l'un et l'autre sont liés par le contrat de mandat. Tel est le cas du chirurgien utilisant les services d'un anesthésiste. Bien entendu, deux médecins peuvent être liés par un contrat de travail, lorsque l'un est salarié par l'autre, ce dernier étant tenu de payer les charges sociales ad hoc et les cotisations paritaires du point de vue de la LPP.

Où se situe la frontière entre mandat et contrat de travail? Lorsque le collaborateur du médecin indépendant déploie une activité identique pour d'autres médecins ou lorsque le praticien indépendant doit faire appel à celui-ci chaque fois qu'une tâche nécessite son intervention, il faut considérer que le contrat de mandat lie les parties. Au contraire, lorsque la personne travaille exclusivement ou à titre principal pour le médecin et qu'elle est véritablement subordonnée à ce dernier, c'est un contrat de travail qui régit leurs relations.

L'importance de la qualification des rapports liant les parties ne présente pas qu'un aspect de théorie juridique. Il faut en effet savoir que les règles appliquées au mandat sont fondamentalement différentes de celles découlant du contrat de travail. Dans le premier cas, le médecin ne doit pas cotiser socialement pour l'autre partie. En outre, chacun pourra résilier le contrat les liant à quelque moment que ce soit et sans délai (le cas de la réparation pour un contrat de mandat résilié en temps inopportun doit être mis en exergue). En revanche, le contrat de travail implique de nombreux droits et obligations pour chaque partie, dont certains feront l'objet de cet article.

Il ne faut pourtant pas espérer pouvoir convenir d'un mandat, lorsque les éléments du contrat de travail existent dans les faits. Un travailleur sous contrat de mandat doit conserver son indépendance par rapport à son mandant, ce qui n'est pas le cas d'un employé face à son patron. Les autorités compétentes en matière fiscale et d'AVS ne reconnaissent en général pas un indépendant ne travaillant que pour une personne; elles le considéreront alors comme employé, quand bien même les parties se sont liées par un contrat de mandat. Devant une juridiction civile également, un «mandataire» ne fournissant des prestations que pour un médecin sera assurément considéré comme un employé, nonobstant l'éventuelle signature d'un contrat de mandat entre les parties. Le médecin devra donc subir les différentes obligations lui incombant en raison du contrat de travail. Il est utile de rappeler que ce contrat, à l'instar du mandat, peut être conclu même en l'absence d'un document écrit et signé.

\section{Le contrat type de travail pour assistante médicale}

Comme il en est fait mention ci-dessus, il existe un formulaire pré-imprimé, communément utilisé par les médecins indépendants en vue de la concrétisation des rapports de travail mis en place avec une assistante. Ce contrat est le fruit de négociations menées entre la FMH et les organisations de défense des salariés FSAAM et SVA.

Malgré l'existence de ce formulaire, qui déroge sur certains points à la réglementation du Code des obligations, notamment en raison des particularités de la profession d'assistante médicale (horaires notamment), le contrat n'est pour- 
tant pas une convention collective de travail de la branche qui revêtirait un caractère obligatoire. Dès lors, médecin et assistante médicale ont la liberté de convenir d'un autre contrat de travail qui devra cependant respecter les dispositions légales impératives.

Le contrat type est fréquemment utilisé en raison de sa grande commodité (il est presque exhaustif) et du fait qu'il est adapté aux circonstances découlant du travail au sein d'un cabinet médical.

\section{Le salaire}

Le médecin peut rémunérer l'assistante médicale de deux manières distinctes: par un salaire prédéfini (en général payé mensuellement) ou en convenant d'un salaire à l'heure.

Le principe du salaire «mensuel» est généralement appliqué pour les assistantes médicales travaillant à plein temps. Au contraire, le taux horaire est fréquemment utilisé pour une employée à temps réduit, sachant que le taux convenu par contrat sera bien souvent dépassé, ce que le salaire par heure permet de prendre en compte aisément en ce qui concerne la rétribution (heures supplémentaires).

Il est à relever qu'il est concevable de convenir d'un salaire «mensuel» pour une assistante médicale à temps partiel. Il faudra cependant tenir compte de ses éventuelles heures supplémentaires qui devront être payées ou compensées par un congé d'une durée équivalente.

En ce qui concerne le droit aux vacances, de quatre semaines au minimum en droit suisse, il est inclus financièrement dans la rétribution lorsque l'employée est rémunérée sur la base d'un salaire prédéfini (puisqu'elle est payée pendant ses vacances). En revanche, dans le cas du salaire par heure de travail, le droit aux vacances fait l'objet d'un versement de salaire complémentaire à l'employée fixé forfaitairement par un pourcentage en général $(8,33 \%$ pour quatre semaines de vacances accordées; 10,64\% si cinq semaines ont été convenues, etc.).

\section{Le secret professionnel}

Le médecin est soumis à une stricte confidentialité quant aux informations qu'il détient sur ses patients. Par contrat de travail, ses employées sont soumises au même secret médical.

La violation de celui-ci peut entraîner des conséquences pénales. L'article 321, alinéa premier du Code pénal, nomme expressément la profession de médecin et les conséquences de la violation de ce devoir et précise que ce dernier incombe de manière identique aux assistantes médicales.

\section{Les absences pour maladie ou accident de I'assistante médicale et le cas de la grossesse}

Lorsque l'employée est malade ou accidentée sans sa faute, le médecin doit lui verser son salaire. Pendant la première année de service, l'employeur doit le salaire pendant trois semaines au minimum. Ensuite, la loi ne précise pas la durée du versement, mais les tribunaux estiment le plus souvent que deux mois de salaire au moins devraient être versés entre la deuxième et la quatrième année de service, ou encore quatre mois dès la dixième année, etc. Ces montants sont en principe des minima et peuvent être augmentés.

Une fois que le médecin a dépassé le délai d'obligation de verser le salaire, il continue certes à compter l'assistante médicale parmi ses employées, mais ne doit plus verser son salaire.

Bien souvent, le médecin a conclu un contrat d'assurance tendant à la compensation de la perte de gain en cas d'absence de l'employée pour cause de maladie ou d'accident. Dès lors, cette assurance, si elle respecte les critères légaux et contractuels, prendra en charge le versement des sommes dues à la collaboratrice absente en lieu et place du médecin.

Il est à relever que les femmes enceintes sont protégées par des dispositions particulières de la loi, de caractère impératif. Les accouchées ne peuvent pas être occupées pendant les huit semaines suivant l'accouchement. Sur simple avis, les femmes enceintes peuvent être dispensées de travail. D'autres règles sont encore posées obligeant l'employeur à certains comportements, comme par exemple ne pas exposer l'employée à des dangers potentiels pour sa santé ou celle de son enfant. En ce qui concerne le versement du salaire, les règles appliquées sont celles concernant l'absence pour maladie ou accident exposées ci-dessus. Il faut signaler que l'allocation de maternité pour les mères exerçant une activité lucrative entrera en vigueur au $1^{\text {er }}$ juillet 2005.

\section{Le licenciement de I'assistante médicale}

Lorsque le médecin désire se séparer d'une employée, il doit respecter les injonctions légales en la matière. 
Le licenciement ordinaire doit être fait de la manière suivante: la décision doit être motivée par écrit si l'employée le demande. En outre, le médecin doit lui communiquer son congé, lorsque le contrat de travail a été conclu pour une durée indéterminée pour la fin d'un mois, avec un délai de:

- un mois lors de la première année de service (sous réserve de la période d'essai);

- deux mois entre la deuxième et la neuvième année de service;

- trois mois ultérieurement.

Bien entendu, si des délais plus longs ont été prévus par les parties, ceux-ci feront alors foi.

Sans entrer dans le détail, il faut relever qu'il peut exister une cause de congé extraordinaire (justes motifs) qui peut être donné avec effet immédiat par l'employeur, lorsque l'employée a commis une ou plusieurs fautes graves. Les motifs d'un tel licenciement doivent être considérés avec réserve.

L'arrêt de travail pour accident ou maladie a une influence sur le congé. En effet, lorsque l'assistante médicale est empêchée sans sa faute de travailler, elle est protégée temporairement de tout licenciement, sauf celui avec effet immédiat. L'employée est protégée du congé pendant trente jours durant la première année de service, quatre-vingt-dix jours entre la deuxième et la cinquième année et cent quatre-vingt dès la sixième. Si le licenciement ordinaire est signifié à la collaboratrice pendant le délai de protection, celui-ci est nul.

Après le délai de protection, l'employeur peut notifier la résiliation du contrat de travail à l'employée, de façon normale.

Il faut encore souligner que lorsque l'assistante médicale est dans l'incapacité de travailler après résiliation de son contrat de travail, mais pendant le délai de congé mentionné ci-dessus, il y aura une suspension du congé tant que dure l'empêchement. Les obligations pécuniaires liées à la maladie ou à l'accident exposées ci-avant devront s'appliquer. En outre, le contrat de travail continuera à déployer ses effets tant que l'incapacité de travail dure, même après la fin de l'obligation de verser le salaire. Le délai de congé ne pourra courir à nouveau qu'à partir de la fin de la maladie ou de l'accident, sauf si la collaboratrice devient bénéficiaire de prestations de l'assurance-invalidité, ce qui aura pour effet de faire cesser le contrat de travail.

L'empêchement de travailler doit être prouvé par la production d'un certificat de maladie, sauf si, dans certaines circonstances, un tel document ne peut être délivré.

\section{Conclusion}

Les rapports entre médecin et employée sont régis de manière stricte par le droit suisse et par les accords entre les parties au contrat de travail.

Si le contrat offre de nombreux droits au médecin, des obligations importantes s'imposent également à lui, notamment en matière salariale.

Il doit être relevé que les conflits entre employeurs et salariés sont de plus en plus fréquemment portés devant les instances judiciaires, sans doute en raison d'un changement de moralité des employés, parfois causé par le comportement des employeurs à leur égard et sans doute aussi parce que l'accès au tribunal est en général gratuit.

Pour éviter autant que possible une comparution devant une cour de justice dans le cadre d'un litige avec un employé, il est important que le médecin s'assure que tout le personnel soit au bénéfice d'un contrat de travail écrit, complet et actualisé. 